\title{
Group visits improved concordance with American Diabetes Association practice guidelines in type 2 diabetes
}

Clancy DE, Cope DW, Magruder KM, et al. Evaluating concordance to American Diabetes Association standards of care for type 2 diabetes through group visits in an uninsured or inadequately insured patient population. Diabetes Care 2003;26:2032-6.

\section{$Q$ In uninsured or inadequately insured patients with uncontrolled type 2 diabetes, does healthcare delivery through group visits promote concordance with American Diabetes Association (ADA) standards of care?}

\section{METHODS}

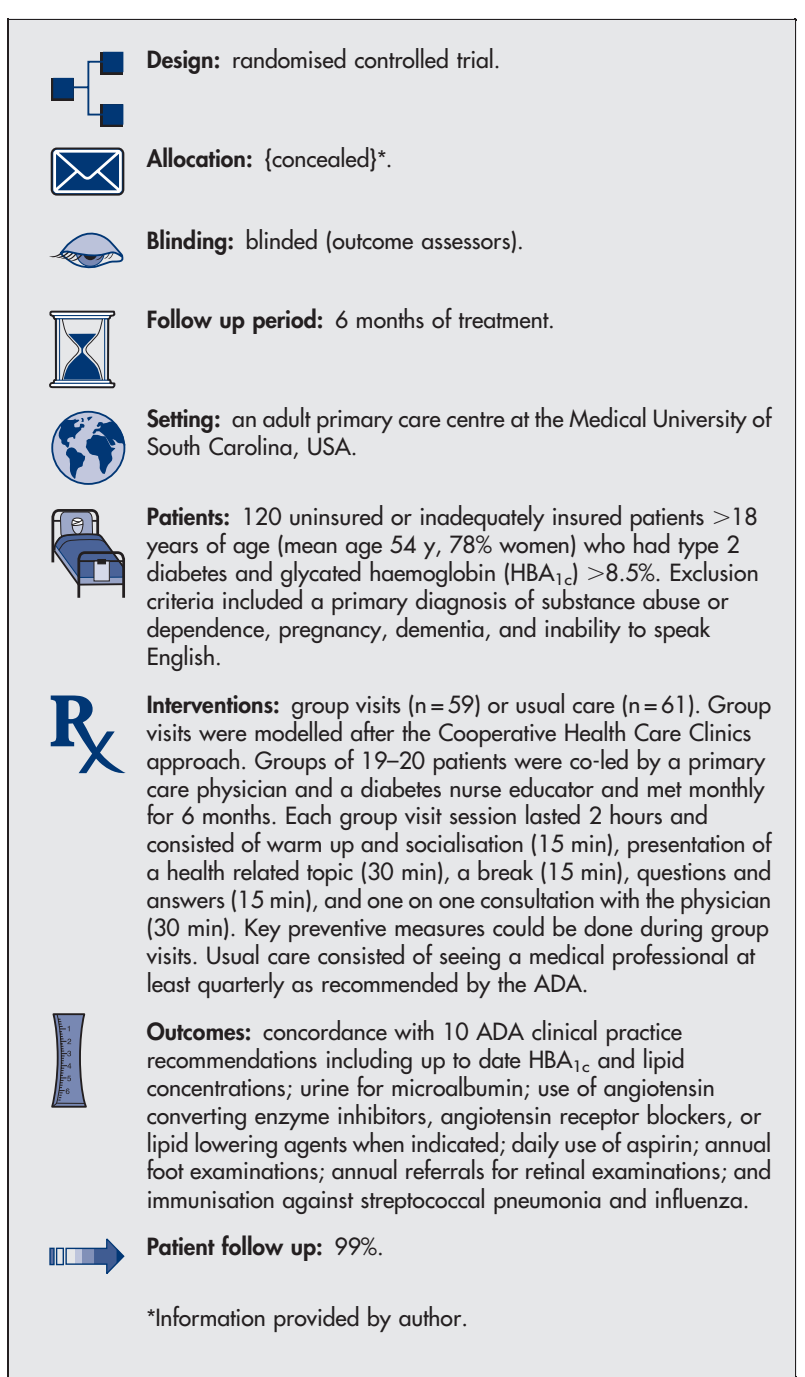

For correspondence: Dr D E Clancy, Medical University of South Carolina, Charleston, SC, USA. clancyd@musc.edu

Sources of funding: Robert Wood Johnson Foundation and the Agency for Healthcare Research and Quality.

\section{MAIN RESULTS}

Analysis was by intention to treat. The mean total number of ADA clinical practice recommendations met per patient was greater in the intervention group than in the usual care group $(8.75 v 7.22$, $\mathrm{p}<0.001$ ). More patients in the intervention group had $\geqslant 8$ of the 10 recommendations addressed (table). The groups did not differ for actual $\mathrm{HBA}_{\mathrm{lc}}$ concentrations or lipid profiles.

\section{CONCLUSION}

In uninsured or inadequately insured patients with uncontrolled type 2 diabetes, healthcare delivery through group visits was more effective than usual care for promoting concordance with American Diabetes Association standards of care.

\section{Commentary}

$\Lambda$ Ithough several studies have explored diabetes education in groups, few have examined group care. The study by Clancy et al provides evidence that group visits for people with type 2 diabetes may have advantages over individual consultations. A significant improvement in achieving standards of care was observed. There were trends, but no statistically significant differences towards improvements in metabolic or lipid control.

A similar study by Trento et al, ${ }^{1}$ with 4 years of follow up, reported stabilised glycaemic control, decreased body mass index, increased high density lipoprotein cholesterol as well as improved knowledge of diabetes, quality of life, and healthcare behaviours in people with type 2 diabetes managed by systematic group education. These results are clinically significant because the usual course of type 2 diabetes is progressive deterioration in biomedical and other outcomes. Perhaps the 6 month follow up of the study by Clancy et al was not long enough to see such clinical improvement.

Strengths of the study by Clancy et al included a low drop out rate and intention to treat analysis; however, most of the patients were women, which may limit generalisability of the findings. The intervention group had increased costs. However, Trento et al concluded that preventing deterioration of glycaemic control and quality of life without increasing pharmacological treatment was cost-effective. ${ }^{1}$ Further work is needed to replicate these studies in different care delivery systems and with longer follow up. However, diabetes care professionals, particularly those in primary care, should consider using groups to provide education and care for people with type 2 diabetes because it may be both more cost efficient and effective than individual consultations.

Maggie Watkinson, RGN, MSc Taunton and Somerset National Health Service Trust Taunton, UK

1 Trento M, Passera P, Bajardi $M$, et al. Lifestyle intervention by group care prevents deterioration of Type II diabetes: a 4-year randomized controlled clinical trial. Diabetologia 2002;45:1231-9.

Group visits $v$ usual care in uncontrolled type 2 diabetes at 6 months*

\begin{tabular}{lllll}
\hline Outcome & Group visits & Usual care & RBI (95\% Cl) & NNT (CI) \\
\hline Patients with $\geqslant 8$ of 10 process of care indicators addressed & $86 \%$ & $47 \%$ & $85 \%$ (42 to 153) & 3 (2 to 5) \\
\hline
\end{tabular}

*Abbreviations defined in glossary; RBI, NNT, and $\mathrm{Cl}$ calculated from data in article. 